\title{
Expression of ERCC1, RRM1, TUBB3 in correlation with apoptosis repressor ARC, DNA mismatch repair proteins and p53 in liver metastasis of colorectal cancer
}

\author{
CSABA TÓTH ${ }^{1}$, FARKAS SÜKÖSD ${ }^{2}$, ERZSÉBET VALICSEK ${ }^{3}$, ESTHER HERPEL $^{1,4}$, PETER SCHIRMACHER $^{1}$, \\ MARCUS RENNER ${ }^{1}$, CHRISTOPH MADER ${ }^{1}$, LÁSZLÓ TISZLAVICZ ${ }^{2}$ and JÖRG KRIEGSMANN ${ }^{5}$ \\ ${ }^{1}$ Institute of Pathology, University Hospital Heidelberg, D-69120 Heidelberg, Germany; \\ Departments of ${ }^{2}$ Pathology and ${ }^{3}$ Oncotherapy, University of Szeged, H-6720 Szeged, Hungary; \\ ${ }^{4}$ Tissue Bank of the National Center for Tumor Diseases (NCT), D-69120 Heidelberg; \\ ${ }^{5}$ MVZ for Histology, Cytology and Molecular Diagnostics, Trier, D-54296 Trier, Germany
}

Received December 12, 2016; Accepted August 25, 2017

DOI: $10.3892 / \mathrm{ijmm} .2017 .3136$

\begin{abstract}
Liver metastasis in colorectal cancer is common and the primary treatment is chemotherapy. To date, there is no routinely used test in clinical practice to predict the effectiveness of conventional chemotherapy. Therefore, biomarkers with predictive value for conventional chemotherapy would be of considerable benefit in treatment planning. We analysed three proteins [excision repair cross-complementing 1 (ERCC1), ribonucleoside-diphosphate reductase 1 (RRM1) and class III $\beta$-tubulin (TUBB3)] in colorectal cancer liver metastasis. We used tissue microarray slides with 101 liver metastasis samples, stained for ERCC1, RRM1 and TUBB3 and established scoring systems (fitted for tissue microarray) for each protein. In statistical analysis, we compared the expression of ERCC1, RRM1 and TUBB3 to mismatch proteins (MLH1, MSH2, MSH6 and PMS2), p53 and to apoptosis repressor protein (ARC). Statistically significant correlations were found between ERCC1, TUBB3 and MLH1, MSH2 and RRM1 and MSH2, MSH6. Noteworthy, our analysis revealed a strong significant correlation between cytoplasmic ARC expression and RRM1, TUBB3 ( $\mathrm{p}=0.000$ and $\mathrm{p}=0.001$, respectively), implying an additional role of TUBB3 and RRM1 not only in therapy resistance, but also in the apoptotic machinery. Our data strengthens the importance of ERCC1, TUBB3 and RRM1 in the prediction of chemotherapy effectiveness and suggest new functional connections in DNA repair, microtubule network and apoptotic signaling (i.e. ARC protein). In conclusion, we showed the importance and need of predictive
\end{abstract}

Correspondence to: Dr Csaba Tóth, ${ }^{5}$ Present address: MVZ for Histology, Cytology and Molecular Diagnostics, Trier, 5 Max Planck Street, D-54296 Trier, Germany

E-mail: c.toth@patho-trier.de

Key words: excision repair cross-complementing 1, class III $\beta$-tubulin, apoptosis repressor protein, ribonucleoside-diphosphate reductase 1, colorectal cancer, liver metastasis, MMR proteins biomarkers in metastasized colorectal cancer and pointed out the relevance not only of single predictive markers but also of their interactions with other known and newly explored relations between different signaling pathways.

\section{Introduction}

Colorectal cancer (CRC) remains a leading cause of cancerassociated deaths worldwide with an incidence of over one million newly diagnosed cases per year. Despite intensive research and therapeutic efforts, the mortality rate of CRC is $\sim 40-50 \%$ (1). Furthermore, the rate of metastatic cases is high (2). Drug resistance is responsible for poor prognosis in many cancer types (3). Thus, to identify proteins which may have predictive value is of importance not only in metastasized CRC, but also in other advanced epithelial cancers. In this regard, the deregulation of DNA damage repair systems (i.e. mismatch repair) represents an important aspect, since it contributes to the resistance of cancer cells to conventional chemotherapy.

One further repair protein is excision repair crosscomplementing 1 (ERCC1), which is also implicated in therapy resistance. ERCC1 is a structure specific DNA repair endonuclease responsible for 5' incision (5'-endonuclease), a key enzyme in the nucleotide excision repair (NER) pathway and is essential for repair of platinum-DNA adducts, and is thus associated with therapy resistance to platinum-containing compounds $(3,4)$. NER is responsible for repair of DNA damages caused by oxidative and alkylating agents (3). ERCC1 was suggested as a promising marker in CRC (4). ERCC1-overexpressing cancer cells are thought to be more resistant to platinum-based chemotherapy. Increased ERCC1 mRNA levels were found to be associated with resistance to platinum-based chemotherapy (i.e. ovarian, gastric, cervical, colorectal and non-small cell lung cancer) suggesting that platinum-paclitaxel chemotherapy would be more effective in ERCC1-negative cancer (3). It is known that ERCC1 protein expression, estimated by immunohistochemistry, is an independent prognostic factor for progression-free and overall 
survival in NSCLC patients treated with platinum-based chemotherapy (5). Similar data could be achieved in CRC (6). In several trials on CRC, the ERCC1 expression level was proposed as a candidate marker for predicting the efficacy of oxaliplatin therapy for metastatic patients. In stage III colon cancer, ERCC1 expression is strongly predictive in the selection of patients which will benefit from additional oxaliplatin to 5-fluorouracil (5-FU) therapy (7).

Ribonucleoside-diphosphate reductase 1 (RRM1) gene encodes the regulatory subunit of ribonucleotide reductase enzyme. Ribonucleotide reductase, composed of regulatory subunit RRM1 and the catalytic subunit RRM2, is a crucial enzyme in new DNA synthesis, catalysing the biosynthesis of deoxyribonucleotides from the corresponding ribonucleotides (8). RRM1 is a key molecule for gemcitabine efficacy and is also involved in tumor progression. High RRM1 expression in tumor tissue predicts significantly better prognosis while only patients with low RRM1 benefit from gemcitabine therapy. In turn, overexpression of RRM1 protein is strongly associated with gemcitabine resistance (8). RRM1 expression was also reported to correlate with the tumorigenic and metastatic potential in lung cancer (8).

The cell cytoskeleton is built up from microtubules, microfilaments and intermediate filaments. Various changes in the microtubule network have been identified in a wide range of cancers, i.e. altered expression of tubulin isotypes, alterations in tubulin posttranslational modifications and changes in the expression of microtubule-associated proteins (MAPs) (9). Class III $\beta$-tubulin (TUBB3) is one of the main microtubule (MT) proteins and is primarily expressed in neurons and Sertoli cells in the testis $(10,11)$. In lung cancer, the TUBB3 protein expression level was found to have no correlation with age, gender, smoking status or recurrence pattern or response rate to chemotherapy. The response rate in TUBB3-positive cases was $18 \%$, while the rate was $27 \%$ in negative cases (no significant differences could be detected) (5). High TUBB3 expression levels are associated with poor prognosis in many epithelial cancers. Additionally, TUBB3 has been suggested to take part in disease aggressiveness by acting as a survival factor for cancer cells (12). In colorectal adenomas, TUBB3 expression can be detected in up to $100 \%$ of high-grade dysplasia. Expression of TUBB3 was found to have no association with grade of dysplasia or other clinical data in preneoplastic lesions of CRC but was associated with Dukes' stage (13). TUBB3 overexpression in colon cancer cells may contribute to a higher stability of the microtubular network which may explain the lower activity of anti-microtubule agents (14). In addition, high TUBB3 expression levels were localized to the invasive edge in CRC; positive TUBB3 staining was observed in all cases, yet this was most prominent at the invasive front with the presence of tumor budding (12). This preferential localization of TUBB3 at the invasive margin raises the possibility that changes in tubulin isotypes can modulate the invasive activity of cancer cells. Microtubules are indispensable for the directional migration of cells. Tubulins, the major constituent protein of microtubules, are built up from heterodimers of $\alpha$ and $\beta$ subunits (12). It is believed that tumor buds consist of migrating cells and TUBB3 expression in these cells is linked to their motility. Furthermore, TUBB3 is expressed in a variety of tumors, particularly in those that are aggressive and likely to metastasize, and were found to be more resistant to several chemotherapy regimens (i.e. estramustine, Taxol, paclitaxel and docetaxel) (12). As we previously demonstrated, MMR protein expression is correlated with the expression of the apoptosis repressor protein apoptosis repressor protein (ARC). It is known that overexpression of ERCC1, RRM1 and TUBB3 is linked to therapeutic resistance against therapeutic regimens, which is also found in advanced (stage IV) CRC (15). In this context, we investigated the expression trend of ERCC1, RRM1 and TUBB3 proteins and their correlation to ARC protein expression, which is known to be upregulated in $\mathrm{CRC}$ and associated with therapeutic resistance inhibiting both extrinsic and intrinsic apoptotic signaling.

\section{Materials and methods}

Tissue samples. Paraffin-embedded surgical specimens of liver metastasis of CRC were selected from the archives of the Institute of Pathology at the University Hospital of Heidelberg. One hundred patients (64 male, 37 female; mean age 62 years) were included. None of the patients had received neo-adjuvant chemotherapy. Tissue samples were fixed in neutral-buffered formalin and embedded in paraffin. Paraffin sections were cut (4 $\mu \mathrm{m})$ and examined on coated slide glass for immunohistochemistry. Further data, such as age, sex, size and number of metastases were collected from histological studies. Tissue samples were provided by the Tissue Bank of the National Center for Tumor Diseases (NCT, Heidelberg, Germany) in accordance with the regulations of the tissue bank and the approval of the Ethics Committee of Heidelberg University according to ethical standards formulated in the Declaration of Helsinki 1975 (revised in 1983).

Tissue microarray. Tissue microarray (TMA) blocks were obtained from paraffin-embedded human liver specimens with a tissue microarrayer (Beecher Instruments, Sun Prairie, WI, USA). From each case, two cores of tumor tissue with a diameter size of $1.6 \mathrm{~mm}$ were punched and for orientation of the TMA slides two muscle cores were used. Muscle punches served also as positive controls for ARC immunostaining.

Immunohistochemistry. Four-micrometer-thick slides were obtained from the TMA. Slides were then deparaffinised according to standard protocol by xylene, and dehydrated with 95-96\% ethanol, 70\% ethanol and distilled water. All slides were stained simultaneously using a computer-controlled autostainer (Ventana BenchMark Ultra, Ventana Medical Systems, Inc., Tucson, AZ, USA). Then primary antibodies were used: ERCC1 (8F1, Neomarkers; dilution 1:100), RRM1 (Protein Tech Europe; dilution 1:200) and TUBB3 (Tuj-1/TubIII/4G3, Covalab; dilution 1:2,000). Primary antibodies were incubated according to routine staining protocols for diagnostic purpose. To detect immunoreactions, Ultraview Universal DAB dectection kit (Ventana Medical Systems, Inc.) and 3,3'-diaminobenzidine were used. A counterstain was performed with hematoxylin and bluing reagent and all slides were covered. For MMR proteins, p53 and ARC, the staining methods were performed as previously published (16).

Evaluation of immunohistochemistry. For semi-quantitative assessment of staining intensity, we adjusted a previously 
Table I. Results of the immunohistochemistry for ERCC1, RRM1 and TUBB3.

\begin{tabular}{lcccr}
\hline Proteins & $\begin{array}{c}\text { Immunoreactive score 0 } \\
\mathrm{n}(\%)\end{array}$ & $\begin{array}{c}\text { Immunoreactive score 1 } \\
\mathrm{n}(\%)\end{array}$ & $\begin{array}{c}\text { Immunoreactive score 2 } \\
\mathrm{n}(\%)\end{array}$ & $\begin{array}{c}\text { Valid cases } \\
\mathrm{n}(\%)\end{array}$ \\
\hline ERCC1 & $28(29.8)$ & $29(30.8)$ & $37(39.4)$ & $94(100)$ \\
RRM1 & $11(11.6)$ & $33(34.7)$ & $51(53.7)$ & $95(100)$ \\
TUBB3 & $35(35)$ & $52(52)$ & $13(13)$ & $100(100)$ \\
\hline
\end{tabular}

ERCC1, excision repair cross-complementing 1; RRM1, ribonucleoside-diphosphate reductase 1; TUBB3, class III $\beta$-tubulin.

published scoring system for each protein and fitted to TMA dots $(17,18)$. ERCC1 and RRM1 immunostainings were scored using a three-graded scale: score 0 , no expression detectable or faint partial expression in $<10 \%$ of the tumor cells; score 1 , weak to moderate expression of the entire tumor tissue; score 2 , strong positivity in the entire tumor tissue.

For TUBB3, a modified three-graded score was established: score 0 , no expression detectable or faint partial expression in $<10 \%$ of the tumor cells; score 1, diffuse and strong positive staining associated to invasive front and tumor budding, central tumor regions negative or with weaker intensity than at the invasive front; score 2, strong positivity in the entire tumor tissue.

For MSI proteins, the staining was evaluated according to Bethesda guidelines (19). Immunostaining for p53 was scored using a three-graded scale: score 0 , weak staining in $<10 \%$ of the tumor cells, score 1 , moderate staining in up to $75 \%$ of the tumor cells and score 2 , strong nuclear staining in $>75 \%$ of the tumor cells. The results of MMR, p53 and ARC immunohistochemistry for this collective have already been published (16).

The immunostained tissue microarray sections were evaluated and scored under a light microscope independently by two pathologists in a blinded manner. Discordant cases were reviewed and re-evaluated based on a consensus opinion.

Statistical analysis. The statistical analyses were performed with SAS software (SAS Institute, Cary, NC, USA). Associations between clinical data, ARC, MMR proteins, ERCC1, TUBB3 and RRM1 were estimated by Pearson's correlation and linear regression test. The statistical significance was set at $\mathrm{p}<0.05$ and $\mathrm{p}<0.01$.

\section{Results}

Distribution of ERCC1, RRM1 and TUBB3 protein expression in the collective. The results of the immunohistochemistry for ERCC1, RRM1 and TUBB3 are listed in Table I. For ERCC1 we found $29.8 \%$ of the cases to be negative (score 0 ). Positive ERCC1 staining was detected in $70.2 \%$ of the cases (score 1, $30.8 \%$ and score 2, 39.4\%). For RRM1, the distribution was different. Only 11 cases out of 95 valid cases $(11.6 \%)$ were found to be negative (score 0). Eighty-four cases (88.4\%) showed positive staining for RRM1, 51 cases showed a high expression level (score 2, 53.7\%).

TUBB3 staining showed an interesting distribution. Most of the cases showed pronounced positivity at the invasive margin (52\%). Thirty-five cases (35\%) had negative staining and only $13 \%$ had a diffuse positive staining reaction for
$\mathbf{A}$

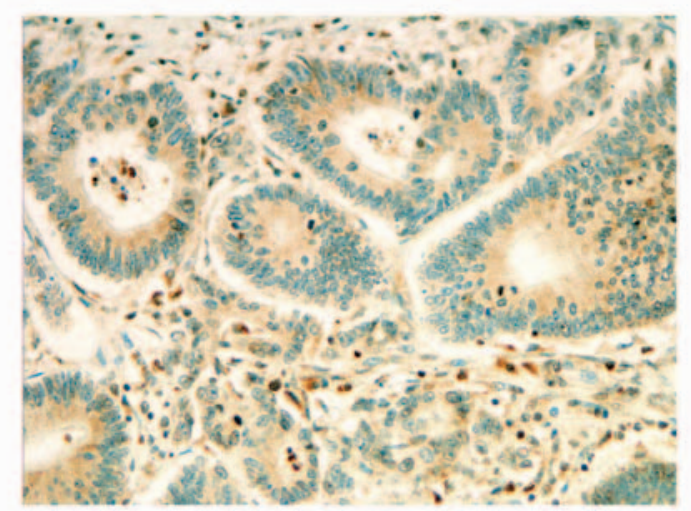

B

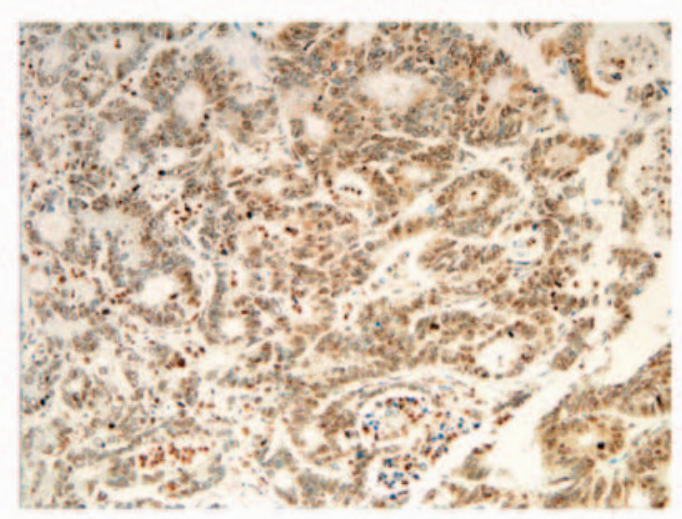

C

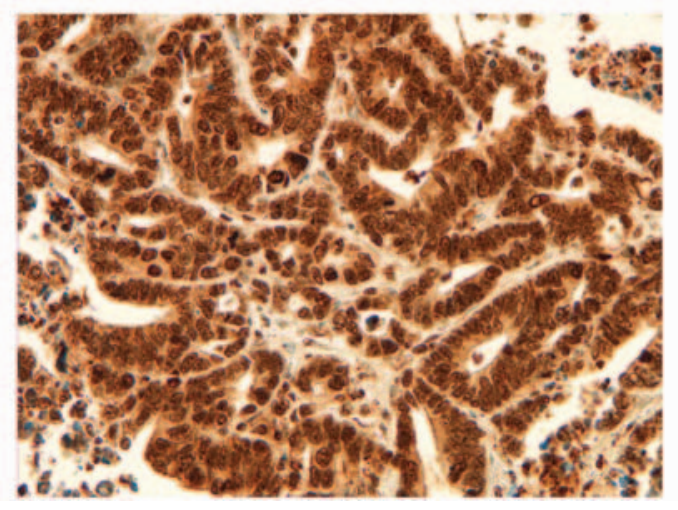

Figure 1. Representative examples of the expression of excision repair crosscomplementing 1 (ERCC1) protein in colorectal liver metastasis. (A) Score 0, no expression detectable or faint partial expression in $<10 \%$ of the tumor cells; (B) score 1, weak to moderate expression of the entire tumor tissue; (C) score 2, strong positivity in the entire tumor tissue; magnification, $\mathrm{x} 400$.

TUBB3. Representative images of the staining scores for ERCC1, RRM1 and TUBB3 are shown in Figs. 1-3. 
A

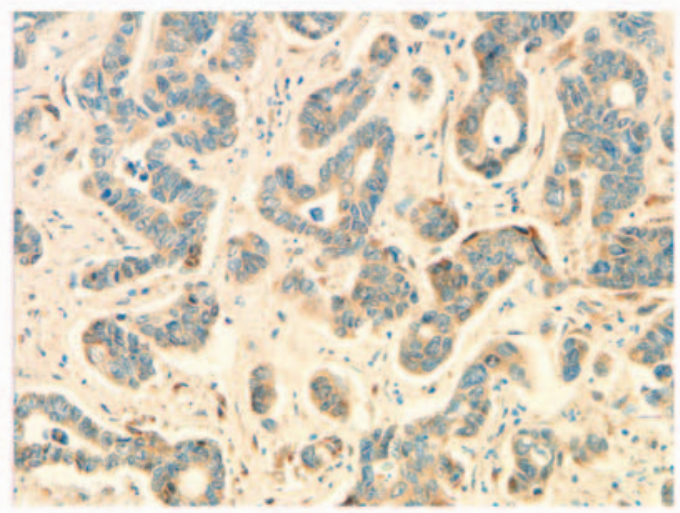

B

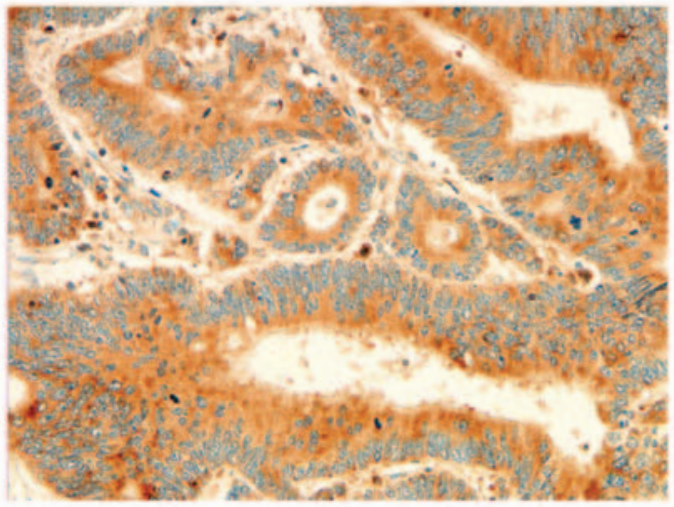

C

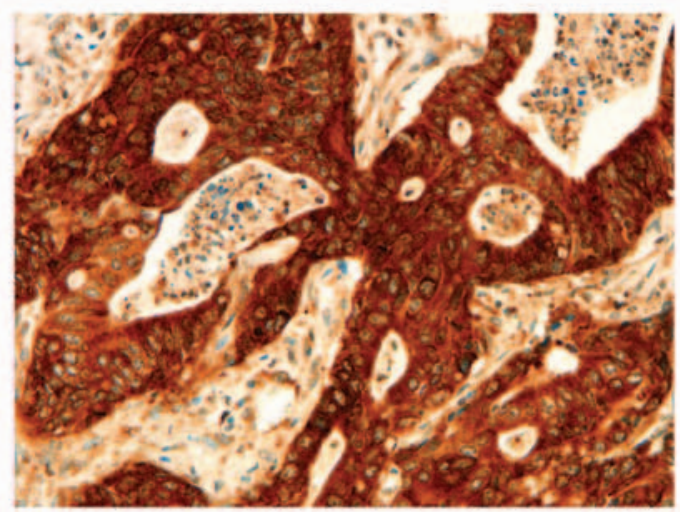

Figure 2. Representative examples of the expression of ribonucleoside-diphosphate reductase 1 (RRM1) protein in colorectal liver metastasis. (A) Score 0, no expression detectable or faint partial expression in $<10 \%$ of the tumor cells; (B) score 1, weak to moderate expression of the entire tumor tissue; (C) score 2, strong positivity in the entire tumor tissue; magnification, $\mathrm{x} 400$.

Statistically significant correlation between ERCC1, RRM1, TUBB3 and MMR proteins, but not with $p 53$. Regarding MMR proteins we found statistically significant correlations between MMR proteins and ERCC1, RRM1 and TUBB3. In turn, none of the three markers demonstrated a correlation with the p53 expression level. MLH1 and MSH2 proteins showed a positive statistically significant correlation with ERCC1 ( $<<0.000$ and $\mathrm{p}=0.008$, respectively). This means that loss of MLH1 and $\mathrm{MSH} 2$ is associated with lower expression or loss of ERCC1 in colorectal liver metastasis. A similar correlation was detected between MSH2, MSH6 and RRM1 ( $\mathrm{p}=0.005$ for MSH2 and $\mathrm{p}=0.011$ for MSH6). Higher RRM1 expression levels were detected at intact expression of MSH2 and MSH6.

Notably, TUBB3 expression showed a strong positive correlation with MLH1 and MSH2 $(\mathrm{p}=0.019$ and $\mathrm{p}=0.012$,
$\mathbf{A}$

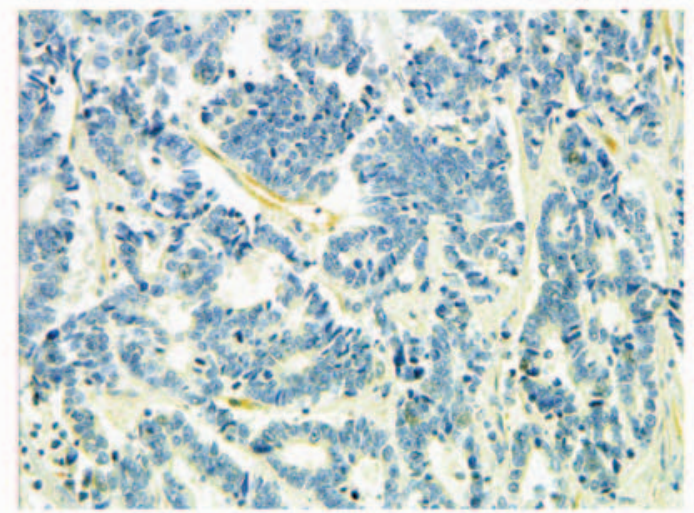

B

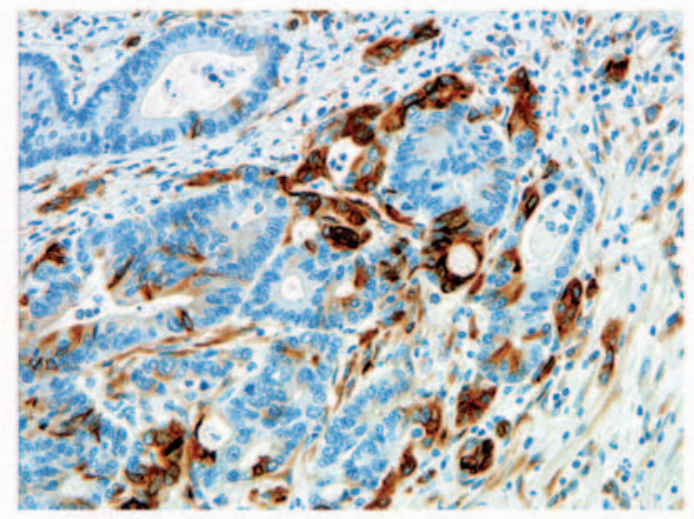

C

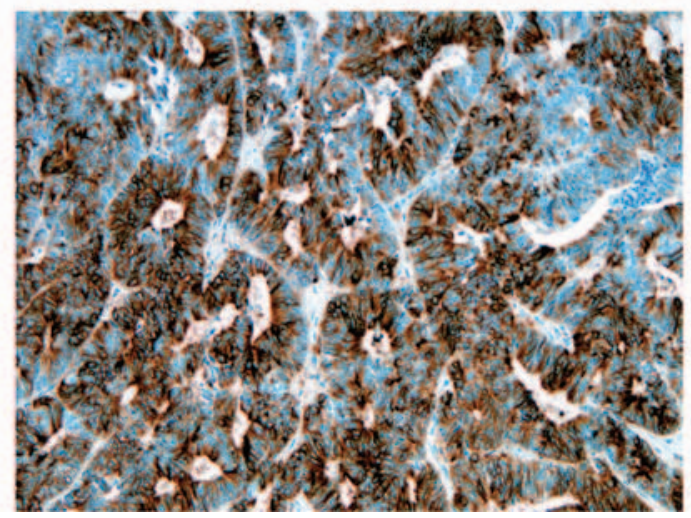

Figure 3. Representative examples of the expression of class III $\beta$-tubulin (TUBB3) protein in colorectal liver metastasis. (A) Score 0, no expression detectable or faint partial expression in $<10 \%$ of the tumor cells; (B) score 1 , diffuse and strong positive staining associated to invasive front and tumor budding, central tumor regions negative or with weaker intensity then at the invasive front; (C) score 2 , strong positivity in the entire tumor tissue; magnification, $\mathrm{x} 400$.

respectively). The detailed correlations are documented in Table II.

Cytoplasmic ARC staining intensity is strongly correlated with TUBB3 and RRM1 expression levels. In negative RRM1 cases, the ARC cytoplasmic expression was also low (score 0/1) (6/10,60\%). Cases with moderate RRM1 expression (score 1) also had in the majority of cases a low level of ARC expression (19/33, 57.6\%). Fourteen of 33 cases (42.4\%) with moderate RRM1 expression had a high level of cytoplasmic ARC (score 2/3). Cancers expressing RRM1 at high levels (score 2, 51 cases) showed, in the majority of cases, elevated cytoplasmic ARC levels [low ARC level in only 12 cases 
Table II. Statistical correlations between ERCC1, RRM1 and TUBB3 with mismatch repair proteins and p53.

\begin{tabular}{|c|c|c|c|c|c|}
\hline Protein & MLH1 & MSH2 & MSH6 & PMS2 & $\mathrm{p} 53$ \\
\hline \multicolumn{6}{|l|}{ ERCC1 } \\
\hline Correlation coefficient & $0.541^{\mathrm{a}}$ & $0.273^{\mathrm{a}}$ & 0.186 & 0.168 & 0.164 \\
\hline Significance (2-sided) & 0.000 & 0.008 & 0.072 & 0.106 & 0.116 \\
\hline No. of valid cases & 71 & 94 & 94 & 94 & 93 \\
\hline \multicolumn{6}{|l|}{ RRM1 } \\
\hline Correlation coefficient & 0.104 & $0.283^{\mathrm{a}}$ & $0.261^{b}$ & 0.198 & 0.146 \\
\hline Significance (2-sided) & 0.387 & 0.005 & 0.011 & 0.055 & 0.160 \\
\hline No. of valid cases & 71 & 95 & 95 & 95 & 94 \\
\hline \multicolumn{6}{|l|}{ TUBB3 } \\
\hline Correlation coefficient & $0.271^{b}$ & $0.252^{\mathrm{b}}$ & 0.025 & 0.033 & 0.035 \\
\hline Significance (2-sided) & 0.019 & 0.012 & 0.808 & 0.748 & 0.736 \\
\hline No. of valid cases & 75 & 99 & 99 & 99 & 98 \\
\hline
\end{tabular}

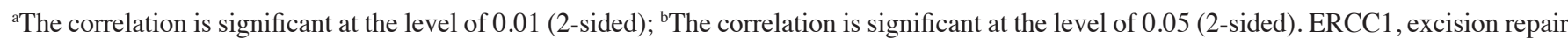
cross-complementing 1; RRM1, ribonucleoside-diphosphate reductase 1; TUBB3, class III $\beta$-tubulin.

Table III. Results of the statistical analysis between apoptosis repressor ARC protein and ERCC1, RRM1 and TUBB3.

\begin{tabular}{lcc}
\hline Protein & $\begin{array}{c}\text { Cytoplasmic ARC } \\
\text { expression }\end{array}$ & $\begin{array}{c}\text { Nuclear ARC } \\
\text { expression }\end{array}$ \\
\hline ERCC1 & & \\
Correlation coefficient & -0.053 & -0.020 \\
Significance (2-sided) & 0.613 & 0.851 \\
Number of valid cases & 93 & 93 \\
RRM1 & & 0.147 \\
Correlation coefficient & $\mathbf{0 . 3 7 8}$ & 0.156 \\
Significance (2-sided) & $\mathbf{0 . 0 0 0}$ & 94 \\
Number of valid cases & $\mathbf{9 4}$ & -0.048 \\
TUBB3 & & 0.641 \\
Correlation coefficient & $\mathbf{0 . 3 2 3}$ & 98 \\
Significance (2-sided) & $\mathbf{0 . 0 0 1}$ & $\mathbf{9 8}$ \\
Number of valid cases & $\mathbf{9 8}$ & \\
\hline
\end{tabular}

${ }^{a}$ The correlation is significant at the level of 0.01 (2-sided); ${ }^{\text {b The cor- }}$ relation is significant at the level of 0.05 (2-sided). ERCC1, excision repair cross-complementing 1; RRM1, ribonucleoside-diphosphate reductase 1 ; TUBB3, class III $\beta$-tubulin.

(12/51, 23.53\%), high ARC level in 39 cases (39/51, 76.47\%)]. In conclusion, a high level of RRM1 expression in most of the cases occurred simultaneously with elevated, high level cytoplasmic ARC protein expression (score $2 / 3$ in $76.47 \%$ of the valid cases). Cytoplasmic ARC protein expression showed a positive, statistically significant correlation with RRM1 expression levels $(\mathrm{p}<0.000)$. Ninty-eight cases were valid for both proteins (ARC cytoplasmic and TUBB3). In TUBB3 negative cases (score 0), cytoplasmic expression of ARC was detected in 15 cases $(15 / 35,42.85 \%)$. In TUBB 3 score 1 cases, it was even higher $(35 / 51,68.62 \%)$. In strongly diffuse positive
Table IV. Results of the statistical analysis between ERCC1, RRM1 and TUBB3 and clinical data.

\begin{tabular}{lcccc}
\hline Protein & Age & Sex & $\begin{array}{c}\text { Tumor } \\
\text { grade }\end{array}$ & $\begin{array}{c}\text { No. of } \\
\text { metastases }\end{array}$ \\
\hline ERCC1 & & & & \\
Correlation coefficient & 0.106 & -0.205 & -0.010 & -0.030 \\
Significance (2-sided) & 0.320 & 0.054 & 0.930 & 0.781 \\
No. of valid cases & 90 & 89 & 82 & 86 \\
RRM1 & & & & \\
Correlation coefficient & 0.004 & 0.001 & 0.151 & 0.027 \\
Significance (2-sided) & 0.971 & 0.995 & 0.174 & 0.803 \\
No. of valid cases & 91 & 90 & 83 & 87 \\
TUBB3 & & & & \\
Correlation coefficient & $\mathbf{- 0 . 2 6 9}$ & -0.139 & $\mathbf{0 . 2 1 3}$ & 0.026 \\
Significance (2-sided) & 0.008 & 0.178 & 0.047 & 0.807 \\
No. of valid cases & 96 & 95 & 87 & 92 \\
\hline
\end{tabular}

${ }^{\text {a }}$ The correlation is significant at the level of 0.01 (2-sided); ${ }^{\text {b }}$ The correlation is significant at the level of 0.05 (2-sided). ERCC1, excision repair cross-complementing 1; RRM1, ribonucleoside-diphosphate reductase 1; TUBB3, class III $\beta$-tubulin.

TUBB3 cases, the highest cytoplasmic ARC expression was found $(9 / 12,75 \%)$. We found a progressive staining intensity for cytoplasmic ARC regarding TUBB3 status. This association was also significant $(\mathrm{p}<0.001)$. The distribution of the statistical results is listed in Table III.

Correlations between ERCC1, RRM1, TUBB3 and clinical data. Concerning clinical parameters such as age, sex of the patients, grade of the tumor and the number and size of metastases, there was no significant correlation with ERCC1 or RRM1 expression (Table IV). Regarding patient age, there 
was a strong negative correlation with TUBB3 expression level $(\mathrm{p}=0.008)$. In addition, the TUBB3 expression level was also positively associated with tumor grade $(\mathrm{p}=0.047)$ and TUBB3 expression was also correlated with the RRM1 expression level $(\mathrm{p}=0.022)$.

\section{Discussion}

Apoptotic signaling is one of the most important processes in therapeutic resistance. In addition to known regulatory proteins, there are many others, which can influence the apoptotic process, and some can thus enhance or inhibit therapeutic effects. ERCC1, RRM1 and TUBB3 are known to have therapeutic predictive value in the current therapy of metastasized CRC $(3,5,6)$. In the present study, we investigated the expression levels of ERCC1, RRM1 and TUBB3 in liver metastasis of CRC and analysed their associations to sex, age, tumor grade, mucin production, tumor size and number of metastases. Furthermore, we investigated their correlation to MMR proteins, p53 and apoptosis repressor ARC.

In our collective, ERCC1 protein loss was detected in onethird of the cases $(29.8 \%)$. A proportion of $70.2 \%$ showed a positive reaction and score 2 (strong nuclear expression) was confirmed in $39.4 \%$ of all valid cases. In another study, which investigated stage III CRC, ERCC1 and MSI levels were found to be positive in 55 and 17\%, respectively (7). According to literature data, score 2 cases do not benefit from platinumbased chemotherapy. On the other hand, one-third of cases (loss of ERCC1) have a better prognosis following platinum-based chemotherapy (15). Cases with moderate ERCC1 expression (score 1), in our opinion, require further investigation. It must be evaluated in functional studies, whether a mild loss in ERCC1 expression is enough to sensitize cancer cells to platinum-based chemotherapy. According to our scoring system, we detected certain negative and positive cases, thus allowing a prediction for platinum-based chemotherapy in two-thirds of the patients after a single immunohistochemistry.

We found a statistically significant positive correlation between ERCC1 and MLH1, MSH2 (p=0.000 and p=0.008, respectively). The frequent loss of ERCC1 and MLH1 both could be explained by methylation. Similar correlations were found in mesothelioma (20). In NSCLC, ERCC1 nuclear staining was noted in $45.59 \%$ of the cases, and TUBB3 cytoplasmic staining was noted in $65.44 \%$ of the cases detected. ERCC1 and TUBB3 double-negative cases exhibited a better therapeutic response to platinum-based therapy (3). We found 11 cases (11.7\% of valid cases) as double-negative for ERCC1 and TUBB3. Analogous to other epithelial neoplasia, $11.7 \%$ of the metastatic cases could benefit much more from platinumbased therapy, than the others. Negative expression of ERCC1 and TUBB3 was found to be associated with a significantly higher response rate, longer median progression-free survival and overall survival after platinum-paclitaxel treatment (3). One study found that patients with advanced CRC with high expression of ERCC1 are not indicated for oxaliplatin-based chemotherapy (17), whereas patients with low levels of ERCC1 expression have been reported to have an improved response and a longer overall survival in metastatic CRC treated with FOLFOX combination (18). In this study, a low level of ERCC1 was detected in $90 \%$ of the cases (18). One study, in accordance with other study results, demonstrated the potential utility of ERCC1 expression as a prognostic and possibly predictive biomarker in metastasized CRC. It was able to identify a population with poor prognosis, as well as a population with a markedly high response rate to FOLFOX combination therapy (18). The cases were evaluated according to a pre-established cut-off for ERCC1 (17). As noted, the expression levels of ERCC1 are not always consistent with sensitivity to platinum-based treatment. We assume that the cut-off level was set too high and suggest to define more than only two groups (positive and negative). Therefore, we propose the use of large prospective or retrospective studies with standard chemotherapy to analyse the expression of predictive markers, such as ERCC1, RRM1 or TUBB3 (and its interaction partners) to set a cut-off for cases, which can benefit from a therapeutic regimen. Too high or too low cut-off levels result in subsets of patients, who may suffer from long-term toxicity with no benefit of treatment, and patient groups that do not receive the optimal therapy regimen.

RRM1 overexpression is associated with gemcitabine resistance. RRM1 is a key molecule for gemcitabine efficacy and is also involved in tumor progression. High RRM1 expression in tumor tissue predicts significantly better prognosis, whereas only patients with low RRM1 benefit from gemcitabine therapy. In turn, overexpression of RRM1 protein is strongly associated with gemcitabine resistance (8). RRM1 expression was also reported to correlate with tumorigenic and metastatic potential in lung cancer (8). According to our data, only $11.6 \%$ of the cases with loss of RRM1 expression had a positive response to gemcitabine. In other words, patients with high RRM1 protein expression in tumor tissue should be treated with alternative drugs, i.e. oxaliplatin, 5-FU and leucovorin (CONKO-003) instead of gemcitabine (8). As mentioned above, score 1 cases need further functional analysis and lower RRM1 activity may also be sufficient not to overcome gemcitabine-induced DNA damage leading to the death of tumor cells.

Loss of MSH2 and MSH6 expression is associated with lower levels of RRM1 protein ( $\mathrm{p}=0.005$ and $\mathrm{p}=0.011$, respectively). For this association the following mechanisms could be responsible: i) RRM1 is a key enzyme in the synthesis of new DNA, thus defected MMR proteins, i.e. MSH2 and MSH6 lead to DNA damage which can downregulate the new DNA synthesis, leading to cell cycle arrest. One possible connection between RRM1 and the MMR system is through DNA damage. It leads to cell cycle arrest, which results in lower RRM1 expression level or ii) there is a possible connection through transforming growth factor- $\beta$ (TGF- $\beta$ ); in normal cells TGF- $\beta$ can activate the MSH2 promoter (through Smad p53 dependent mechanism), whereas at the posttranscriptional level, miR-21 induced by TGF- $\beta$ targets MSH2 transcript and suppresses its expression. In contrast, in cancer cells p53 is inactivated and miR-21 is overexpressed, thus TGF- $\beta$ fails to activate the $\mathrm{MSH} 2$ promoter resulting in genomic instability (21).

Cytoplasmic ARC protein expression showed a positive, statistically significant correlation with RRM1 expression levels ( $\mathrm{p}<0.000)$. Which mechanism leads to higher RRM1 expression with ARC overexpression or in turn how ARC expression can induce RRM1 overexpression should be elucidated in functional studies, but it is known that RRM1overexpressing cells have an increased level of apoptosis (22), 


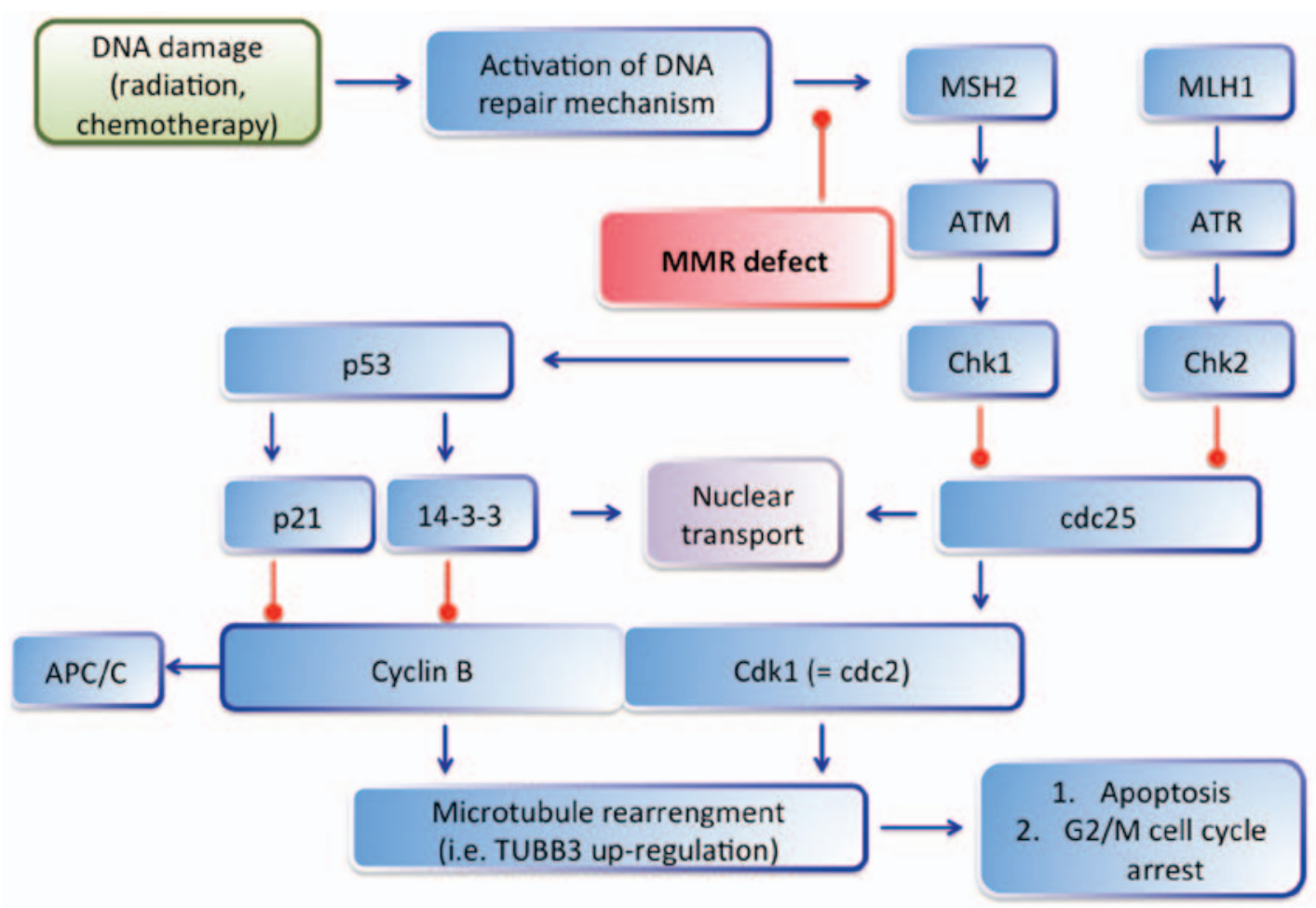

Figure 4. Schematic presentation of DNA damage-induced apoptosis and the possible role of the microtubule system.

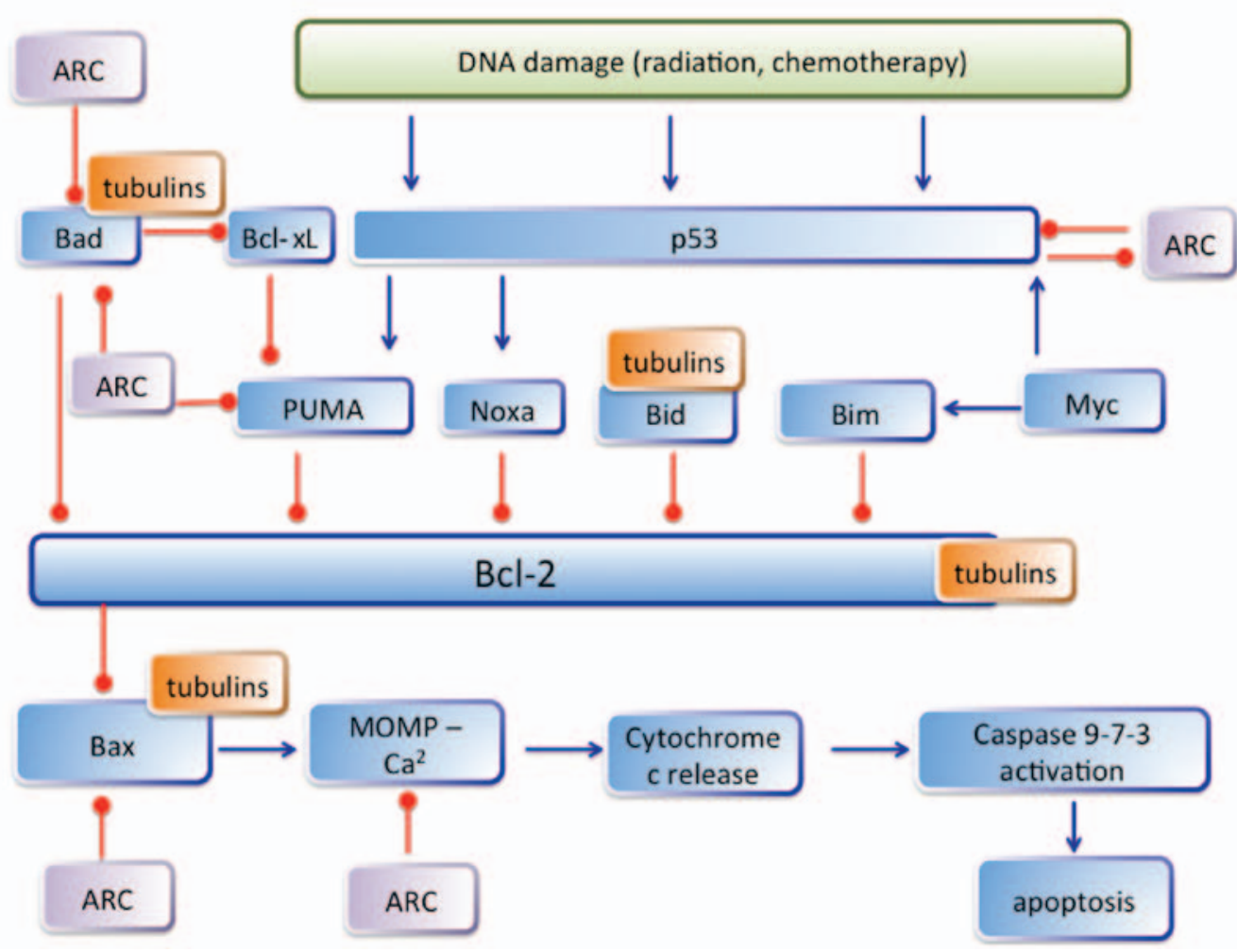

Figure 5. Interactions between tubulins and apoptotic signaling proteins including apoptosis repressor protein (ARC).

thus it is possible that a certain overexpression level can induce apoptotic signaling, which in turn induces ARC expression to suppress apoptosis induction. This possibility is further strengthened by the positive correlation between RRM1 and TUBB3 found in our collective $(\mathrm{p}=0.022)$.
The main staining pattern for TUBB3 was expression at the invasive front, similar to primary CRC studied previously (12). TUBB3 expression was not detected in $35 \%$ of the valid cases. These cases are potential candidates for taxanebased chemotherapy with highly predicted response. In our 
collective, we found a statistically significant correlation between MLH1, MSH2 and TUBB3 ( $\mathrm{p}=0.019$ and $\mathrm{p}=0.012$, respectively), which further strengthens the evidence of the regulatory role of mismatch repair proteins in apoptosis. In the case of sufficient MLH1 and MSH2 expression, TUBB3 is significantly highly expressed to suppress the activities of MLH1 and MSH2.

These results can indicate that a defected MMR system would induce TUBB3 overexpression leading to MT rearrangement, which can influence apoptosis (i.e. activating pro-apoptotic signaling proteins). Microtubules (MTs) have an important role in apoptosis, i.e. survivin is believed to regulate apoptosis by controlling microtubule polymerization. Thus, the disruption of normal MT function (either increasing or decreasing MT length) may trigger apoptosis. MT system (and thus TUBB3) has an important role in the regulation of DNA damage-induced apoptosis. DNA damage (i.e. $\gamma$-radiation) induces $\alpha$-, $\beta$ - and $\gamma$-tubulin production and polymerization, and stimulates MT reorganization (23). One explanation is that DNA damage through cyclin B1 and cdc2 kinase activation leads to tubulin polymerization and to release of apoptosis (23). The possible connection between TUBB3, DNA damage and mismatch repair are depicted in Fig. 4. After DNA damage, the ATM/ATR signaling pathway is activated and phosphorylates (and activates) Chk1 and Chk2, which subsequently phosphorylate cdc25 (23). Phosporylated cdc25 is sequestered in the cytoplasm by 14-3-3 proteins, which hinder the activation of cyclin B1/Cdk1 complex by cdc25 resulting in $\mathrm{G} 2 / \mathrm{M}$ cell cycle arrest. In the case of MMR loss (i.e. in our cases, the loss of MLH1 and MSH2) the ATM/ATR system cannot be activated by MMR proteins and finally do not lead to cell cycle arrest. Consequently, microtubule rearrangement and TUBB3 upregulation is lacking. This correlation between MLH1/MSH2 and TUBB3 was statistically significant in our collective ( $\mathrm{p}=0.019$ and $\mathrm{p}=0.012$, respectively).

Furthermore, MLH1 and MSH2 are responsible for resistance to cisplatin or methylating agents. The defective MMR system cannot recognize the cisplatin-induced DNA damage resulting in cell survival and therapeutic resistance $(24,25)$. Taken together, cases with defected MMR system (microsatellite instable cancer) and with a high expression level of TUBB3 are potentially resistant not only to taxanes, but also to platinum-based therapy. Thus, we favor, in the case of MSI, immunohistochemical testing also for TUBB3 to exclude taxane resistance. The interactions between DNA repair systems, MT and apoptotic proteins (i.e. ARC) should be further investigated to elucidate the resistance mechanism of tumor cells and the survival regulatory mechanism.

In addition and alternatively to the above mentioned mechanisms, the statistically significant correlation between TUBB3 and cytoplasmic ARC expression $(\mathrm{p}=0.001)$ can also be explained. Survival feedback mechanism can induce ARC expression to suppress pro-apoptotic signaling, thus cancer cells can survive despite of DNA damage (i.e. microsatellite instability). In our previous study, we found a strong correlation between ARC expression level and MSH2 status (16). TUBB3 overexpression can stabilize the MT system and make cancer cells resistant against anti-microtubule agents. Direct interaction between tubulin with several members of the Bcl-2 family has been described. Bcl-2, Bid and Bad were found to inhibit the assembly, whereas Bak and Bax promote tubulin polymerization. Thereby, tubulin is localized not only in the cytoplasm, but also binds to mitochondria (associated with VDAC in mitochondrial membrane). Both pro- and antiapoptotic proteins bind to tubulin and those of lower affinity are more easily released following a conformational change induced by a ligand. Thus, Bcl-2, Bid and Bad may remain bound, while Bax would be released changing the ratio of free pro- and anti-apoptotic proteins. Furthermore, in the case of TUBB3 overexpression pro- and anti-apoptotic proteins stay bound, but tubulin ligands can change the affinity towards proteases. In addition, Bcl-2 protects against acetylation of tubulin and Bcl-2 is able to normalize the level of acetylated tubulin (26). The interaction between TUBB3 and apoptotic proteins (especially between ARC and TUBB3) seems to be more complex. There are many common interaction partners of ARC and TUBB3 and which protein effects will dominate depends on intracellular circumstances. The known interactions between apoptotic proteins, including ARC and TUBB3 are depicted in Fig. 5. Despite the increasing number of studies that highlight the importance of TUBB3 in tumor cells, its mode of action still needs to be fully determined. It appears that the intrinsic apoptotic pathway is involved as evidenced by increased caspase-3/7 activity (27). Evidence in other cell types suggests that TUBB3 may be part of a cell survival pathway. For instance, its expression level can be modulated by different types of cell stress, i.e. hypoxia (anti-VEGFR therapy) and nutrient deprivation $(28,29)$. To confirm the interaction between ARC and TUBB3, functional studies are needed.

Testing the expression of ERCC1, RRM1 and TUBB3 is crucial and necessary before treatment for gemcitabine, cisplatin and 5-FU. As known, MSI tumors will not benefit from 5-FU treatment and, to this analogy, the testing for ERCC1, RRM1 and TUBB3 before platinum-based, gemcitabine and taxane therapy, respectively. There are a lack of diagnostic tests to determine which chemotherapy regimen offers the greatest chance for response in an individual patient (18). For metastatic $\mathrm{CRC}$, the current treatment paradigm consists of 5-FU-based regimens in combination with either oxaliplatin (FOLFOX) or irinotecan (FOLFIRI), potentially combined with therapy targeting either EGFR or VEGFR inhibitor (30). Large, prospective clinical trials have shown that the response rates for either FOLFOX or FOLFIRI are only approximately $55 \%$ (15). Thus, there is an urgent need for reliable predictive markers before therapeutic decision in metastasized CRC.

In conclusion, we found statistically significant correlations between MMR proteins and ERCC1, RRM1 and TUBB3. Furthermore, we found a statistically significant correlation between the apoptosis repressor protein ARC and RRM1 and TUBB3. Taken together, regarding these proteins, there is a high therapeutic resistance potential in CRC metastasis. Thus we propose to test the known associated predictive proteins, before any therapeutic option is offered. Further functional studies need to declare the exact regulatory mechanism between RRM1, TUBB3 and ARC, as exact relations among these proteins cannot be measured by means of immunohistochemistry alone. The assessment of the abovementioned markers may be a helpful tool to design chemotherapy protocols for CRC liver metastasis and to define patients who may expect a greater clinical benefit. Selection of chemotherapeutic 
drugs according to their predicted efficacy should be a part of future therapeutic decisions and prospective studies. A prospective validation of these markers is warranted.

\section{References}

1. Siegel R, Desantis C and Jemal A: Colorectal cancer statistics, 2014. CA Cancer J Clin 64: 104-117, 2014.

2. Binefa G, Rodríguez-Moranta F, Teule A and Medina-Hayas M: Colorectal cancer: From prevention to personalized medicine. World J Gastroenterol 20: 6786-6808, 2014.

3. Li Z, Qing Y, Guan W, Li M, Peng Y, Zhang S, Xiong Y and Wang D: Predictive value of APE1, BRCA1, ERCC1 and TUBB3 expression in patients with advanced non-small cell lung cancer (NSCLC) receiving first-line platinum-paclitaxel chemotherapy. Cancer Chemother Pharmacol 74: 777-786, 2014.

4. Ruzzo A, Graziano F, Loupakis F, Rulli E, Canestrari E, Santini D, Catalano V, Ficarelli R, Maltese P, Bisonni R, et al: Pharmacogenetic profiling in patients with advanced colorectal cancer treated with first-line FOLFOX-4 chemotherapy. J Clin Oncol 25: 1247-1254, 2007.

5. Azuma K, Sasada T, Kawahara A, Takamori S, Hattori S, Ikeda J, Itoh K, Yamada A, Kage M, Kuwano M, et al: Expression of ERCC1 and class III beta-tubulin in non-small cell lung cancer patients treated with carboplatin and paclitaxel. Lung Cancer 64: 326-333, 2009.

6. Shirota Y, Stoehlmacher J, Brabender J, Xiong YP, Uetake H, Danenberg KD, Groshen S, Tsao-Wei DD, Danenberg PV and Lenz HJ: ERCC1 and thymidylate synthase mRNA levels predict survival for colorectal cancer patients receiving combination oxaliplatin and fluorouracil chemotherapy. J Clin Oncol 19 4298-4304, 2001.

7. Li P, Fang YJ, Li F, Ou QJ, Chen G and Ma G: ERCC1, defective mismatch repair status as predictive biomarkers of survival for stage III colon cancer patients receiving oxaliplatin-based adjuvant chemotherapy. Br J Cancer 108: 1238-1244, 2013.

8. Akita H, Zheng Z, Takeda Y, Kim C, Kittaka N, Kobayashi S, Marubashi S, Takemasa I, Nagano H, Dono K, et al: Significance of RRM1 and ERCC1 expression in resectable pancreatic adenocarcinoma. Oncogene 28: 2903-2909, 2009.

9. Parker AL, Kavallaris M and McCarroll JA: Microtubules and their role in cellular stress in cancer. Front Oncol 4: 153, 2014.

10. Guo J, Qiang M and Ludueña RF: The distribution of $\beta$-tubulin isotypes in cultured neurons from embryonic, newborn, and adult mouse brains. Brain Res 1420: 8-18, 2011.

11. Verdier-Pinard P, Pasquier E, Xiao H, Burd B, Villard C, Lafitte D, Miller LM, Angeletti RH, Horwitz SB and Braguer D: Tubulin proteomics: Towards breaking the code. Anal Biochem 384 197-206, 2009.

12. Portyanko A, Kovalev P, Gorgun J and Cherstvoy E: beta(III)tubulin at the invasive margin of colorectal cancer: Possible link to invasion. Virchows Arch 454: 541-548, 2009.

13. Giarnieri E, De Francesco GP, Carico E, Midiri G, Amanti C, Giacomelli L, Tucci G, Gidaro S, Stroppa I, Gidaro G, et al Alpha- and beta-tubulin expression in rectal cancer development. Anticancer Res 25: 3237-3241, 2005.

14. Carles G, Braguer D, Dumontet C, Bourgarel V, Gonçalves A, Sarrazin M, Rognoni JB and Briand C: Differentiation of human colon cancer cells changes the expression of beta-tubulin isotypes and MAPs. Br J Cancer 80: 1162-1168, 1999.

15. Colucci G, Gebbia V, Paoletti G, Giuliani F, Caruso M, Gebbia N, Cartenì G, Agostara B, Pezzella G, Manzione L, et al; Gruppo Oncologico Dell'Italia Meridionale: Phase III randomized trial of FOLFIRI versus FOLFOX4 in the treatment of advanced colorectal cancer: A multicenter study of the Gruppo Oncologico Dell'Italia Meridionale. J Clin Oncol 23: 4866-4875, 2005.
16. Tóth C, Meinrath J, Herpel E, Derix J, Fries J, Buettner R, Schirmacher $\mathrm{P}$ and Heikaus S: Expression of the apoptosis repressor with caspase recruitment domain (ARC) in liver metastasis of colorectal cancer and its correlation with DNA mismatch repair proteins and p53. J Cancer Res Clin Oncol 142: 927-935, 2016.

17. Grimminger PP, Shi M, Barrett C, Lebwohl D, Danenberg KD, Brabender J, Vigen CL, Danenberg PV, Winder T and Lenz HJ: TS and ERCC-1 mRNA expressions and clinical outcome in patients with metastatic colon cancer in CONFIRM-1 and -2 clinical trials. Pharmacogenomics J 12: 404-411, 2012.

18. Choueiri MB, Shen JP, Gross AM, Huang JK, Ideker T and Fanta P: ERCC1 and TS Expression as Prognostic and Predictive Biomarkers in Metastatic Colon Cancer. PLoS One 10: e0126898, 2015.

19. Umar A, Boland CR, Terdiman JP, Syngal S, de la Chapelle A, Rüschoff J, Fishel R, Lindor NM, Burgart LJ, Hamelin R, et al: Revised Bethesda Guidelines for hereditary nonpolyposis colorectal cancer (Lynch syndrome) and microsatellite instability. J Natl Cancer Inst 96: 261-268, 2004.

20. Ting S1, Mairinger FD, Hager T, Welter S, Eberhardt WE, Wohlschlaeger J, Schmid KW and Christoph DC: ERCC1, MLH1, MSH2, MSH6, and betaIII-tubulin: resistance proteins associated with response and outcome to platinum-based chemotherapy in malignant pleural mesothelioma. Clin Lung Cancer 14: 558-567.e3, 2013.

21. Yu Y, Wang Y, Ren X, Tsuyada A, Li A, Liu LJ and Wang SE: Context-dependent bidirectional regulation of the MutS homolog 2 by transforming growth factor $\beta$ contributes to chemoresistance in breast cancer cells. Mol Cancer Res 8: 1633-1642, 2010.

22. Ohtaka K, Kohya N, Sato K, Kitajima Y, Ide T, Mitsuno M and Miyazaki K: Ribonucleotide reductase subunit M1 is a possible chemoresistance marker to gemcitabine in biliary tract carcinoma. Oncol Rep 20: 279-286, 2008.

23. Porter LA and Lee JM: Alpha-, beta-, and gamma-tubulin polymerization in response to DNA damage. Exp Cell Res 270: 151-158, 2001.

24. Hassen S, Ali N and Chowdhury P: Molecular signaling mechanisms of apoptosis in hereditary non-polyposis colorectal cancer. World J Gastrointest Pathophysiol 3: 71-79, 2012.

25. Martin LP, Hamilton TC and Schilder RJ: Platinum resistance: The role of DNA repair pathways. Clin Cancer Res 14: 1291-1295, 2008.

26. Knipling L and Wolff J: Direct interaction of Bcl-2 proteins with tubulin. Biochem Biophys Res Commun 341: 433-439, 2006.

27. McCarroll JA, Sharbeen G, Liu J, Youkhana J, Goldstein D, McCarthy N, Limbri LF, Dischl D, Ceyhan GO, Erkan M, et al: $\beta$ III-tubulin: A novel mediator of chemoresistance and metastases in pancreatic cancer. Oncotarget 6: 2235-2249, 2015.

28. Raspaglio G, Filippetti F, Prislei S, Penci R, De Maria I, Cicchillitti L, Mozzetti S, Scambia G and Ferlini C: Hypoxia induces class III beta-tubulin gene expression by HIF-1alpha binding to its 3' flanking region. Gene 409: 100-108, 2008.

29. Raspaglio G, De Maria I, Filippetti F, Martinelli E, Zannoni GF, Prislei S, Ferrandina G, Shahabi S, Scambia G and Ferlini C: $\mathrm{HuR}$ regulates beta-tubulin isotype expression in ovarian cancer. Cancer Res 70: 5891-5900, 2010.

30. Van Cutsem E, Cervantes A, Nordlinger B and Arnold D; ESMO Guidelines Working Group: Metastatic colorectal cancer: ESMO Clinical Practice Guidelines for diagnosis, treatment and follow-up. Ann Oncol 25 (Suppl 3): iii1-iii9, 2014. 